\title{
Fast and Robust Parameter Estimation in the Application of Fuzzy Logistic Equations in Population Growth
}

\author{
Nor Atirah Izzah Zulkefli*, Yeak Su Hoe and Normah Maan \\ Department of Mathematical Sciences, Faculty of Science \\ Universiti Teknologi Malaysia, 81310 Johor Bahru, Malaysia \\ *Corresponding author: noratirahizzah@gmail.com
}

Article history

Received: 4 March 2019

Received in revised form: 25 June 2019

Accepted: 3 July 2019

Published online: 1 August 2019

\begin{abstract}
In this paper, extended Runge-Kutta fourth order method for directly solving the fuzzy logistic problem is presented. The extended Runge-Kutta method has lower number of function evaluations, compared with the classical Runge-Kutta method. The numerical robustness of the method in parameter estimation is enhanced via error minimization in predicting growth rate and carrying capacity. The results of fuzzy logistic model with the estimated parameters have been compared with population growth data in Malaysia, which indicate that this method is acceptable to represent or predict the data population. Numerical example is given to illustrate the efficiency of the proposed model. It is concluded that robust parameter estimation technique is efficient in modelling population growth.
\end{abstract}

Keywords Fuzzy Logistic Equations, Population Growth, Parameter Estimation, Robust Gradient Minimization

\section{Mathematics Subject Classification 37M05}

\section{Introduction}

Analytically or computationally intractable likelihoods often occur in the genetics and biology literature. Applications involving populations like population growth models can provide important but limited inferences from count data, such as estimates of population growth rate and the maximum limit value of the population. Parameter estimation is a discipline that provides tools for efficient use of data for aiding in mathematical modeling of phenomena and the estimation of parameters in this paper.

The utilization of these models is limited particularly by imprecise estimates of population size, which reduces the accuracy and precision of parameter estimates [1]. When the parameters of a population model must be estimated from time-series data, it is usual to assume that stochasticity would arise from either measurement error or process error, but not both [2]. A process-error model of population dynamics assumes that variation comes from the population 
growth process (e.g., random birth-death processes, environmentally driven variation in survival), and that observations are made without error. Meanwhile, a measurement-error model assumes that changes in population size are deterministic, and that measurements of population size are made with errors. Measurement errors can be viewed as noise that obscures the signal of the true population dynamics. If measurement error is significant but ignored, predictions about the future behavior of a population can be grossly in error because the process variation is overestimated [3-5].

In real research programs, new problems lacking empirical data are common, therefore, probability distribution of variables cannot be obtained. In order to deal with these problems, Liu [6] particularly investigated uncertainty theory, and then refined it in Liu [7]. Nowadays, the uncertainty theory has become a new branch of mathematics for modeling nondeterministic phenomena.

Fuzzy differential equations can be used to describe the phenomena that include uncertainty parameter. This study mainly concerns with fuzzy differential equations and logistic equations in population growth model. This paper presents the approaches for incorporating parameter estimation techniques in fuzzy logistic equations through the use of the robust gradient minimization. This paper aims to discuss the model of fuzzy logistic equation subjected to uncertainties in parameter intrinsic growth rate, $G$ and initial population growth, $y_{0}$.

There have been many different methods for solving fuzzy logistic equations, such as explicit Runge-Kutta methods to integrate systems. This study has been conducted with aim to solve fuzzy logistic equations by using extended Runge-Kutta fourth order method. The results have been compared with data population growth model.

The rest of the paper is organized as follows: The Model Description section presents some basic concepts and properties in fuzzy theory in logistic model. The Parameter Estimation section explains the derivation of robust minimization, while the Numerical Solution of the Model section explains numerical method using the extended Runge-Kutta fourth order method. The Numerical Example section gives a numerical example to show the efficiency of the solution of population growth model.

\section{Preliminaries}

Zadeh [8] extended the definition of the characteristic functions by replacing the set $\{0,1\}$ by closed interval $[0,1]$ which is the base to the new definition of fuzzy sets.

Definition 1 [9] Let $Y$ be the universal set with typical element, denoted by $y$. A fuzzy set $A$ in $Y$ is characterized by a membership function $\mu_{A}: Y \rightarrow[0,1]$, with the value $\mu_{A}(y)$ representing the grade of membership of $y$ in $A$.

The set of all fuzzy sets over a set $Y$ will be denoted by $F(Y)$.

Zadeh in his first publication [8] defined the operations for fuzzy sets by generalizing the theoretic operations of crisp sets. Through his research that it is realized that the set theoretic operations intersection, union and complement correspond to the logical operators and, inclusive or and negation.

Definition 2 [10] Let $A$ and $B$ be two fuzzy sets, then: 
1 The intersection of $A$ and $B$ is the fuzzy set $C$ with

$$
C(y)=(A \cap B)(y)=\min \{A(y), B(y)\}=A(y) \wedge B(y) .
$$

2 The union of $A$ and $B$ is the fuzzy set $C$, where

$$
C(y)=(A \cup B)(y)=\max \{A(y), B(y)\}=A(y) \vee B(y) .
$$

3 The complement of $A$ is the fuzzy set $B$, where

$$
B(y)=A^{C}(y)=1-A(y), \forall y \in Y .
$$

4 Difference $(A-B)(y)=\left(A \cap B^{C}\right)(y)=\min \{A(y), 1-B(y)\}$.

5 Equilibrium points $A(y)=A^{C}(y)$.

Now, two fuzzy subsets of a set $Y$ can be compared, as one of them contains the other as follows:

Definition 3 [8] Let $A, B$ be two fuzzy subsets of $Y$, defined as $A \leq B$ to mean $A(y) \leq B(y)$ for all $y \in Y$.

Definition 4 [11] The support of the fuzzy set $A$ is defined by:

$$
\operatorname{supp}(A)=\{y \in Y: A(y)>0\} .
$$

Definition 5 [12] An $\alpha$ - level set of a fuzzy set $A$ of $Y$ is a non-fuzzy set denoted by $A_{\alpha}$ and is defined by:

$$
A_{\alpha}=\left\{\begin{array}{l}
y \in Y: \mu_{\tilde{a}}(y) \geq \alpha, \quad \alpha \in(0,1], \\
\text { closure }\left(\cup_{\alpha \in(0,1]}[\tilde{a}]_{\alpha}\right), \quad \alpha=0 .
\end{array}\right.
$$

\section{Model Description}

The logistic growth model has been introduced to describe population growth with consideration of a self-limitation term that corrects the unlimited growth of the Malthusian model [13].

Malthusian models have the following form:

$$
y(t)=y_{0} e^{G t}
$$

where $y_{0}=y(0)$ is the initial population size and $G$ is the population growth rate, or sometimes called as Malthusian parameter. In real world, with its limited resources, exponential growth cannot continue indefinitely. Exponential growth may occur in environments where there are few individuals and plentiful resources, but when the number of individuals gets large enough, resources will be depleted and the growth rate will slow down [14].

To model the reality of limited resources, population ecologists have developed the logistic growth model. The logistic growth model attempts to model real-world population dynamics by adding carrying capacity, denoted by $K$. 
The logistic growth model can be expressed as Equation (1) as:

$$
y^{\prime}=G y(t)\left(\frac{1-y(t)}{K}\right), y(t)=y_{0} .
$$

Its general solution depends on initial condition, and parameter $G$ is used to define the "fuzzy" solution as an extension of this function following Zadeh's extension principle.

$y(t)$ is $n$-dimensional fuzzy functions of $t$. The function $y^{\prime}(t)$ is a fuzzy derivative of $y(t)$ and $y_{0}$ is a fuzzy number. The fuzzy function $y(t)$ is denoted by $\left[\underline{y}_{\alpha}^{i}(t), \bar{y}_{\alpha}^{i}(t)\right]$. The $\alpha$-cuts of $y(t)$ are follows:

$$
y_{\alpha}^{i}=\left[\underline{y}_{\alpha}^{i}(t), \bar{y}_{\alpha}^{i}(t)\right], i=0, \ldots, n .
$$

The $\alpha$-cuts equations of fuzzy logistic of Equation (2) are defined as follows:

$$
\begin{aligned}
& \underline{y}_{\alpha}^{i}(t)=\min \left\{u \mid u \in\left[\underline{y}_{\alpha}^{i}(t), \bar{y}_{\alpha}^{i}(t)\right]\right\}, \\
& \bar{y}_{\alpha}^{i}(t)=\max \left\{u \mid u \in\left[\underline{y}_{\alpha}^{i}(t), y_{\alpha}^{i}(t)\right]\right\}, \\
& \underline{y}_{\alpha}(0)=\underline{y}_{\alpha 0}, \\
& \bar{y}_{\alpha}(0)=\bar{y}_{\alpha 0} .
\end{aligned}
$$

\section{Parameter Estimation}

The parameters, $G$ and $K$ from Equation (1) are estimated from the minimum of the objective error using robust gradient minimization. From the objective error, $E(G, K)$, the gradient function for $G$ and $K$ are defined as follows:

$$
E(G, K)=\sum_{i=0}^{n}\left(y\left(t_{i}\right)-P\left(t_{i}\right)\right)^{2}=\sum_{i=0}^{n}\left(y_{i}-P_{i}\right)^{2} .
$$

where $y\left(t_{i}\right)$ is a population growth at time $t$, and $P\left(t_{i}\right)$ is the data of population growth model. Equation (4) is differentiated partially with respect to $G$ and $K$, to obtain

$$
\frac{\partial E}{\partial G}=\sum_{i=0}^{n} 2\left(y_{i}-P_{i}\right) \frac{\partial y_{i}}{\partial G}
$$

and

$$
\frac{\partial E}{\partial K}=\sum_{i=0}^{n} 2\left(y_{i}-P_{i}\right) \frac{\partial y_{i}}{\partial K}
$$

Then, Equation (1) is differentiated partially with respect to $G$ and $K$, to obtain

$$
\frac{\partial}{\partial G} \frac{d}{d t} y=y+G \frac{\partial y}{\partial G}-\frac{y^{2}}{K}-G \frac{2 y \frac{\partial y}{\partial G}}{K}
$$

and

$$
\frac{\partial}{\partial K} \frac{d}{d t} y=G \frac{\partial y}{\partial K}-\frac{G y^{2}}{K^{2}}-G \frac{2 y \frac{\partial y}{\partial K}}{K} .
$$


Let $\beta_{1}=\frac{\partial y}{\partial G}$ and $\beta_{2}=\frac{\partial y}{\partial K}$. Then, $\beta_{1}$ and $\beta_{2}$ are substituted into Equations (7) and (8) to obtain

$$
\begin{array}{r}
\frac{d}{d t} \frac{\partial y}{\partial G}=y+G \frac{\partial y}{\partial G}-\frac{y^{2}}{K}-G \frac{2 y \frac{\partial y}{\partial G}}{K} \\
\frac{d}{d t} \beta_{1}=y+G \beta_{1}-\frac{y^{2}}{K}-G \frac{2 y \beta_{1}}{K}
\end{array}
$$

and

$$
\begin{aligned}
\frac{d}{d t} \frac{\partial y}{\partial K} & =G \frac{\partial y}{\partial K}-\frac{G y^{2}}{K^{2}}-G \frac{2 y \frac{\partial y}{\partial K}}{K} \\
\frac{d}{d t} \beta_{2} & =G \beta_{2}-\frac{G y^{2}}{K^{2}}-G \frac{2 y \beta_{2}}{K} .
\end{aligned}
$$

In order to obtain the new value for parameter estimations $G$ and $K$, the gradient of objective function needs to be calculated. The following are derived from Equation (5) obtained

$$
\frac{\partial E}{\partial G}=\sum_{i=0}^{n} 2\left(y_{i}-P_{i}\right) \frac{\partial y_{i}}{\partial G}=\sum_{i=0}^{n} 2\left(y_{i}-P_{i}\right) \beta_{1}^{i},
$$

and from Equation (6),

$$
\frac{\partial E}{\partial K}=\sum_{i=0}^{n} 2\left(y_{i}-P_{i}\right) \frac{\partial y_{i}}{\partial K}=\sum_{i=0}^{n} 2\left(y_{i}-P_{i}\right) \beta_{2}^{i}
$$

The whole minimization from Equation (4) is then solved using gradient based minimization, which is that is conjugate gradient given in Equation (11) and (12). The best value of parameters, $G$ and $K$ are obtained after minimization.

In real population, the growth rate of population in certain countries, which is denoted as parameter in Equation (1), is often imprecise due to the implicit lack of information and the mistakes in measurement process. Therefore, in this paper, fuzzy parameter for $G$ is introduced to design meaningful and realistic models; plus, parameter $K$ is a crisp number. The parameters will be applied to the model and solved using numerical method for the predictions of the country population.

The program code is written in Microsoft Visual $C++$, and Matlab software is used for visualization. The time of calculation has been programmed in accordance to the data.

\section{$5 \quad$ Extended Runge-Kutta Fourth Order Method}

A lot of researches have been done to improve the efficiency of Runge-Kutta method. Increasing the number of terms in the Taylor series expansion is one of the preferable ways to improve the order of accuracy of Runge-Kutta method. In their work, Xinyuan and Jianlin proposed an extended Runge-Kutta-like formulae for integrating autonomous system of ordinary differential equations [15]. Further, as an improvement for solving ordinary differential equations by two and three stages, Rabiei and Ismail developed a third order Improved Runge-Kutta method $[16]$. 
Therefore, the extended Runge-Kutta method has been chosen to solve the problem as discussed in this paper. A family of extended Runge-Kutta method has the form

$$
y_{i+1}=y_{i}+h \sum_{j=1}^{m} b_{j} k_{j}^{(1)}+h^{2} \sum_{j=1}^{m} c_{j} k_{j}^{(2)}
$$

where

$$
\begin{aligned}
& k_{j}^{(1)}=f\left(y_{i}+h \sum_{s=1}^{j-1} a_{j s} k_{s}^{(1)}\right), \\
& k_{j}^{(2)}=f^{\prime}\left(y_{i}+h \sum_{s=1}^{j-1} b_{j s} k_{s}^{(1)}\right), j=1,2, \ldots, m .
\end{aligned}
$$

Obviously, with $c_{j}=0, j=1,2, \cdots, m$ in Equation (13), the method is reduced to classical Runge-Kutta methods, as

$$
y_{i+1}=y_{i}+h \sum_{j=1}^{m} b_{j} k_{j}
$$

where

$$
k_{j}=f\left(y_{i}+h \sum_{s=1}^{j-1} a_{j s} k_{s}^{(1)}\right), \quad j=1,2, \ldots, m .
$$

From Equation (13) and Equation (14), the extended Runge-Kutta methods with $m=3$ have the following form:

$$
y_{i+1}=y_{i}+h\left(b_{1} k_{1}^{(1)}+b_{2} k_{2}^{(1)}+b_{3} k_{3}^{(1)}\right)+h^{2}\left(c_{1} k_{1}^{2}+c_{2} k_{2}^{2}+c_{3} k_{3}^{2}\right),
$$

where

$$
\begin{aligned}
& y_{0}=\alpha \\
& k_{1}^{(1)}=f\left(y_{i}\right) \\
& k_{2}^{(1)}=f\left(y_{i}+h a_{21} k_{1}^{(1)}\right) \\
& k_{3}^{(1)}=f\left(y_{i}+h a_{31} k_{1}^{(1)}+h a_{32} k_{2}^{(1)}\right),
\end{aligned}
$$

and

$$
\begin{aligned}
& k_{1}^{(2)}=f^{\prime}\left(y_{i}\right), \\
& k_{2}^{(2)}=f^{\prime}\left(y_{i}+h b_{21} k_{1}^{(1)}\right), \\
& k_{3}^{(2)}=f^{\prime}\left(y_{i}+h b_{31} k_{1}^{(1)}+h b_{32} k_{2}^{(1)}\right),
\end{aligned}
$$

In order to determine the coefficients of formulae in Equations (17) and (18), Taylor's series expansion is used to compare the terms of order 1,2,3 and 4 with those of the true solution in $\mathrm{Wu}$ and Xia [15]. Accordingly,

$$
b_{1}=1, c_{1}=\frac{1}{6}, c_{3}=\frac{1}{3}, a_{21}=\frac{1}{4}, b_{32}=\frac{1}{2}
$$


Then, the values from Equation (19) are substituted into Equation (17) and(18). Thus, the extended Runge-Kutta fourth order method has the following form:

$$
\begin{array}{rlrl}
y_{i+1} & =y_{i}+h k_{1}^{(1)}+h^{2}\left(\frac{1}{6} k_{2}^{2}+\frac{1}{3} k_{3}^{2}\right), \\
y_{0} & =\alpha & & k_{1}^{(2)}=f^{\prime}\left(y_{i}\right), \\
k_{1}^{(1)} & =f\left(y_{i}\right), & & k_{2}^{(2)}=f^{\prime}\left(y_{i}\right), \\
k_{2}^{(1)} & =f\left(y_{i}+\frac{1}{4} h k_{1}^{(1)}\right), k_{3}^{(2)}=f^{\prime}\left(y_{i}+\frac{1}{2} h k_{2}^{(1)}\right) . \\
k_{3}^{(1)} & =f\left(y_{i}\right), & &
\end{array}
$$

\section{$6 \quad$ Numerical Example}

In this section, the fuzzy logistic equation, Equation (1), with fuzzy initial condition is given as:

$$
\begin{aligned}
\frac{d y}{d t}(t) & =G y(t)\left(1-\frac{y(t)}{K}\right), t \in[0,1] \\
y(0) & =(0.2727473+0.01 \alpha, 0.2927473-0.01 \alpha), 0<\alpha \leq 1 .
\end{aligned}
$$

The parameters, $G$ and $K$ are estimated in the fuzzy logistic model. By minimizing the objective function using conjugate gradient method, the values of parameters $G$ and $K$ are obtained. Next is to proceed the numerical method as proposed in this paper.

In this work, the actual Malaysia (2010-2014) population data as presented in Table 1, from the website (references 17) has been used, with reduced unit from 1 million to 0.1 , normalized time from 0 to 1 .

The obtained results from numerical method and actual population data have been plotted as in Figure 1 and Figure 2. From the graph, we can see that the approximate solution converges to the population data.

\section{Results and Discussion}

Figure 1 presents the percentage of fuzzy width between ERK4 solution and data population; also listed in Table 2.

From Table 2, when $t=0.00$, the percentage of fuzzy width between ERK4 lower solution and data population is $0.037 \%$, while between data population and ERK4 upper solution is $0.035 \%$.

When $t=0.50$, the percentage of fuzzy width between ERK4 lower solution and data population is $0.036 \%$, while between data population and ERK4 upper solution is $0.035 \%$. When $t=0.75$, the percentage of fuzzy width between ERK4 lower solution and data population is $0.036 \%$, while between data population and ERK4 upper solution is $0.034 \%$.

Therefore, the uncertainty region is considered good and acceptable because the percentage of fuzzy width between the initial fuzzy width at $t=0.00$ and the simulation at $t=0.50$ and $t=0.75$ are close. 
Table 1: Normalized Population Data in Malaysia

\begin{tabular}{|c|c|}
\hline Time & Population \\
\hline 0 & 0.282747 \\
\hline 0.02075 & 0.283126 \\
\hline 0.04175 & 0.283504 \\
\hline 0.0625 & 0.283882 \\
\hline 0.08325 & 0.28426 \\
\hline 0.10425 & 0.284638 \\
\hline 0.125 & 0.285017 \\
\hline 0.14575 & 0.285395 \\
\hline 0.16675 & 0.285773 \\
\hline 0.1875 & 0.286151 \\
\hline 0.20825 & 0.28653 \\
\hline 0.22925 & 0.286908 \\
\hline 0.25 & 0.287286 \\
\hline 0.27075 & 0.287662 \\
\hline 0.29175 & 0.288038 \\
\hline 0.3125 & 0.288414 \\
\hline 0.33325 & 0.288791 \\
\hline 0.35425 & 0.289167 \\
\hline 0.375 & 0.289543 \\
\hline 0.39575 & 0.289919 \\
\hline 0.41675 & 0.290295 \\
\hline 0.4375 & 0.290671 \\
\hline 0.45825 & 0.291047 \\
\hline 0.47925 & 0.291423 \\
\hline 0.5 & 0.2918 \\
\hline
\end{tabular}

\begin{tabular}{|l|l|}
\hline Time & Population \\
\hline 0.52075 & 0.292173 \\
\hline 0.54175 & 0.292547 \\
\hline 0.5625 & 0.292921 \\
\hline 0.58325 & 0.293294 \\
\hline 0.60425 & 0.293668 \\
\hline 0.625 & 0.294042 \\
\hline 0.64575 & 0.294415 \\
\hline 0.66675 & 0.294789 \\
\hline 0.6875 & 0.295163 \\
\hline 0.70825 & 0.295537 \\
\hline 0.72925 & 0.29591 \\
\hline 0.75 & 0.296284 \\
\hline 0.77075 & 0.296655 \\
\hline 0.79175 & 0.297026 \\
\hline 0.8125 & 0.297396 \\
\hline 0.83325 & 0.297767 \\
\hline 0.85425 & 0.298138 \\
\hline 0.875 & 0.298509 \\
\hline 0.89575 & 0.29888 \\
\hline 0.91675 & 0.29925 \\
\hline 0.9375 & 0.299621 \\
\hline 0.95825 & 0.299992 \\
\hline 0.97925 & 0.300363 \\
\hline 1 & 0.300734 \\
\hline
\end{tabular}

Table 2: Percentage of Fuzzy Width

\begin{tabular}{|l|l|l|l|l|l|}
\hline Time & $\begin{array}{l}\text { Data } \\
\text { Population }\end{array}$ & $\begin{array}{l}\text { ERK4 } \\
\text { Lower } \\
\text { Solution }\end{array}$ & $\begin{array}{l}\text { ERK4 } \\
\text { Upper } \\
\text { Solution }\end{array}$ & $\begin{array}{l}\text { Percentage of } \\
\text { Fuzzy Width } \\
\text { in Lower } \\
\text { Region }\end{array}$ & $\begin{array}{l}\text { Percentage of } \\
\text { Fuzzy Width } \\
\text { in Upper } \\
\text { Region }\end{array}$ \\
\hline 0.00 & 0.2827473 & 0.2727473 & 0.2927473 & $0.037 \%$ & $0.035 \%$ \\
\hline 0.50 & 0.291799 & 0.281203 & 0.302149 & $0.036 \%$ & $0.035 \%$ \\
\hline 0.75 & 0.296284 & 0.285401 & 0.306789 & $0.036 \%$ & $0.034 \%$ \\
\hline
\end{tabular}




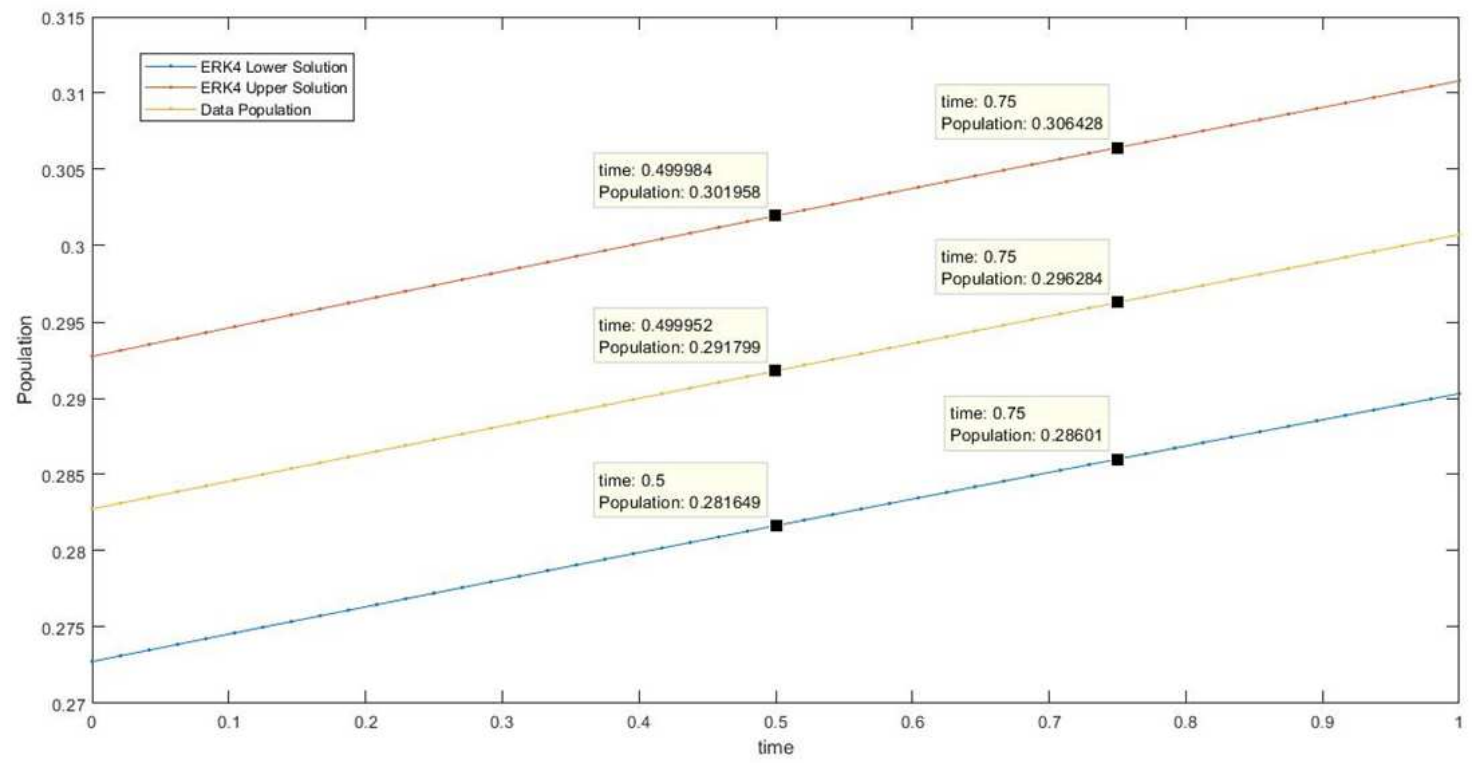

Figure 1: Fuzzy Logistic Equation in 2D

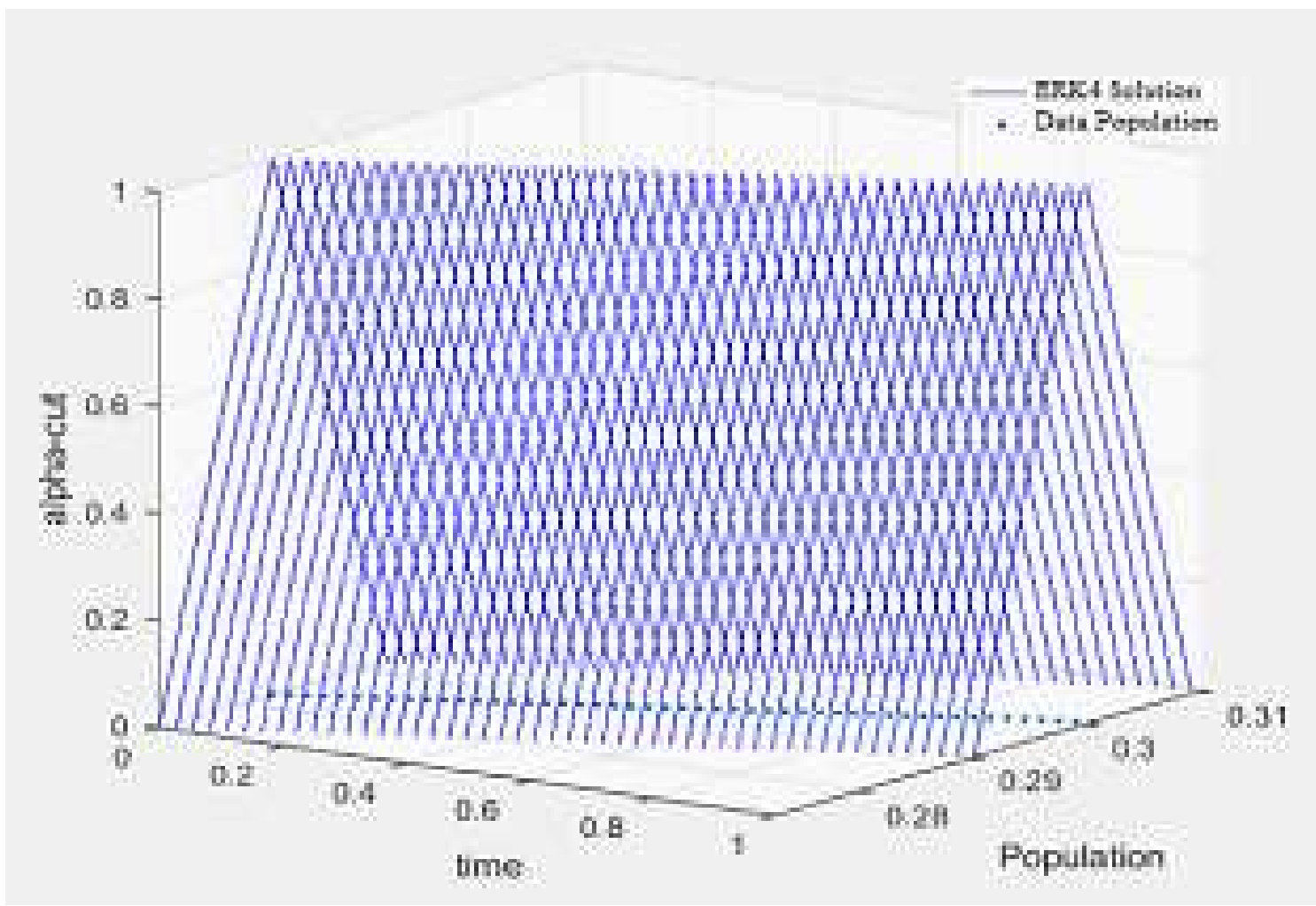

Figure 2: Fuzzy Logistic Equation in 3D 
In previous study [18], we solved this problem using other minimization technique, which is center difference differentiation.

$$
\begin{gathered}
\frac{\partial y_{i}}{\partial G}=\frac{y_{i}(G+\Delta G)-y_{i}(G-\Delta G)}{\Delta G}, \\
\frac{\partial y_{i}}{\partial K}=\frac{y_{i}(K+\Delta K)-y_{i}(K-\Delta K)}{\Delta K}
\end{gathered}
$$

In terms of $\mathrm{CPU}$ time comparison, the ratio of robust minimization and center difference minimization is 0.5337. In computational time, the focus was on how many ODE systems could be solved. Obviously, as in Equation (22), the center difference scheme needs to be calculated 4 times for differential equation, while the robust scheme only has 2 complicated differential equation to be solved. Thus, the ratio should be more than $\frac{2}{4}$ according to our prediction. Therefore, we can conclude that robust approach is more efficient than center difference differentiation.

\section{Conclusion}

This paper has shown how the study of fuzzy differential equations can be motivated using model of populations. This approach permits parameter estimation studies. In this study, robust parameter estimation has been used to estimate the model parameter, and has indeed given better estimation. The robust parameter estimation gives a closer value for parameter $G$ and $K$ to model the population data. The expected result is bounded inside the region of lower and upper solution.

\section{Acknowledgement}

This work was financially supported by the Universiti Teknologi Malaysia under the Research Management Centre - UTM and Research University Grant (GUP) through vote Q.J130000. 2626.13J76, Ministry of Education Malaysia and GE STEM grant with vote number 07397. The authors are thankful for the financial support.

\section{References}

[1] Ludwig, D. Is it meaningful to estimate a probability of extinction? Ecology. 1999. 80: 98-310.

[2] Hilborn, R. and Mangel, M. The Ecological Detective. Princeton, New Jersey, USA: Princeton University Press, 1997.

[3] Dennis, B., Munholland, P. L. and Scott, J. M. Estimation of growth and extinction parameters for endangered species. Ecological Monographs. 1991. 61: 115-143.

[4] Meir, E. and Fagan, W. F. Will observation error and biases ruin the use of simple extinction models? Conservation Biology. 2000. 14: 148-154.

[5] Holmes, E. E. Estimating risks in declining populations with poor data. Proceeding of the National Academy of Sciences (USA). 2001. 98: 5072-5077. 
[6] Liu, B. Uncertainty Theory. 2nd Edn. Berlin: Springer-Verlag. 2007.

[7] Liu, B. Uncertainty Theory: A Branch of Mathematics for Modeling Human Uncertainty. Berlin: Springer-Verlag. 2010.

[8] Zadeh, L. A. Fuzzy sets. Information and Control. 1965. 8(3): 338-353.

[9] Klir, G. J., St Clair, U. and Yuan, B. Fuzzy Set Theory: Foundation and Applications. Prentice-Hall, Inc. 1997.

[10] Zimmermann, H. J. Fuzzy Set Theory. John Wiley \& Sons, Inc. WIREs Comp Stat 2. 2010.

[11] Bede, B. Mathematics of Fuzzy Sets and Fuzzy Logic, STUDFUZZ 295. Berlin Heidelberg: Springer-Verlag. 2013.

[12] Gao, S., Zhang, Z. and Cao, C. Multiplication operation on fuzzy numbers. JSW. 2009. 4(4): 331-338.

[13] Kot, M. Elements of Mathematical Ecology. Cambridge, UK: Cambridge University Press. 2001.

[14] Winch, Donald. Malthus: a Very Short Introduction. Oxford: OUP 2013.

[15] Wu, X. and Xia, J. Extended RungeKutta-like formulae. Application Numerical Math. 2006. 56: 1584-1605.

[16] Rabiei, F. and Ismail, F. Fifth- order Improved Runge-Kutta method for solving ordinary differential equation. Australian Journal of Basic and Applied Sciences. 2012. 6(3): 97-105.

[17] Miguel, B. CIA World Factbook: Accurate as of June 30. In Index Mundi. 2015.

[18] Zulkefli, N. A. I., Yeak, S. H. and Maan, N. The application of fuzzy logistic equations in population growth with parameter estimation via minimization. Malaysian Journal of Fundamental and Applied Sciences. 2017. 13(2): 109-112. 